\title{
NOTAS DE JURISPRUDENCIA DEL TRIBUNAL EUROPEO DE DERECHOS HUMANOS
}

\author{
OMAR BOUAZZA ARIÑO' \\ Universidad Complutense de Madrid \\ Profesor titular de Derecho Administrativo \\ obouazza@der.ucm.es.
}

Cómo citar/Citation

Bouazza Ariño, O. (2020).

Notas de jurisprudencia del Tribunal Europeo de Derechos Humanos.

Revista de Administración Pública, 21 1, 239-254

doi: https://doi.org/10.18042/cepc/rap.21 1.09

I. DERECHO AL RESPETO DE LA VIDA PRIVADA Y FAMILIAR: 1. La Administración deberá realizar una justa ponderación entre los intereses del menor y los de los padres biológicos en situaciones de acogida y adopción. 2. Gestación subrogada. II. DERECHO AL RESPETO DE LOS BIENES: 1. Sanidad animal: no indemnización por la orden de sacrificio de animales enfermos. 2. Vía de hecho. III. DERECHO A LA INSTRUCCIÓN. IV. NON BIS IN IDEM.

1 Este trabajo ha sido realizado en el marco del proyecto de investigación «El reto de la reafirmación del Estado del Bienestar en la protección de los derechos humanos» (DER2015-65524-R), financiado por el Ministerio de Economía y Competitividad (MINECO) y el Fondo Europeo de Desarrollo Regional (FEDER, UE). 


\section{DERECHO AL RESPETO DE LA VIDA PRIVADA Y FAMILIAR}

\section{LA ADMINISTRACIÓN DEBERÁ REALIZAR UNA JUSTA PONDERACIÓN ENTRE LOS INTERESES DEL MENOR Y LOS DE LOS PADRES BIOLÓGICOS EN SITUACIONES DE ACOGIDA Y ADOPCIÓN}

En las sentencias A.S. c. Noruega y Abdi Ibrahim c. Noruega, de 17 de diciembre de 2019, el TEDH resuelve dos casos muy destacados en cuanto a la evolución de las exigencias que se plantean a las Administraciones competentes en materia de servicios sociales en las relaciones de familia. La Administración y los tribunales internos deberán realizar un justo equilibrio, especialmente cuando el interés de los padres entra en conflicto con el de los menores. Solo podrá romperse un vínculo familiar en circunstancias excepcionales. Veamos a continuación los hechos acontecidos, la argumentación y la decisión del TEDH.

Los hijos de las demandantes han sido puestos en acogimiento en contra de su voluntad y, después, entregados en adopción. La demandante en el primer caso es una ciudadana polaca nacida en 1968, mientras que la demandante en el segundo caso es una somalí nacida en 1993.

El hijo de la primera demandante, que nació en 2009, quedó bajo la custodia de la Administración por razones de urgencia. Y después, en 2012, fue acogido en el seno de una familia. En 2014, A.S. solicitó ante el tribunal de distrito poner fin a la situación de acogimiento de su hijo. Su solicitud no solo sería denegada, sino que además se rechazó su petición alternativa de acordar un régimen de visitas. Tampoco se le comunicó la dirección de la familia de acogimiento, como pretendió. El tribunal justificó esta decisión en los problemas de desarrollo del niño. La demandante reconoció que la orden de puesta en acogimiento en 2012 estaba justificada, pero alegó que sus aptitudes maternales habían mejorado tras haber realizado unos cursos específicos. El tribunal observó que los reencuentros entre la demandante y su hijo habían demostrado su incapacidad de empatía con el niño y de tener en cuenta sus necesidades. No daría especial importancia a las alegaciones de la demandante en cuanto a la mejora de sus aptitudes. La demandante recurrió esta decisión sin éxito.

El hijo de la segunda demandante, nacido en Kenia en 2009, antes de llegar a Noruega con su madre, que había obtenido el estatus de refugiada, fue puesto en custodia urgente de la Administración en diciembre de 2010. Más tarde, una familia cristiana acogería al menor, si bien la demandante había indicado que familiares suyos podían cuidar al menor o, en su caso, una familia somalí o musulmana.

La Administración solicitó que la familia de acogimiento fuera autorizada para adoptarlo, lo que comportaba que la madre perdía los derechos referidos a la patria potestad y la prohibición de todo contacto con su hijo. La interesada recurrió. No solicitaba la devolución del niño, ya que había pasado bastante tiempo 
con sus padres de acogida y se había integrado bien, sino un derecho de visita con el fin de que el niño pudiera conservar sus raíces culturales y religiosas.

El tribunal de apelación autorizó la adopción en mayo de 2015. Tuvo en cuenta las cuestiones étnicas, culturales y religiosas que implicaba la adopción del menor por una familia cristiana. La demandante recurrió sin éxito la autorización ante el Tribunal Supremo.

La demandante del primer caso se queja ante el TEDH de las decisiones en base a las cuales las autoridades internas rechazaron poner fin al acogimiento de su hijo en una familia de acogida, reconocerle un derecho de visita y comunicarle la dirección de su hijo.

En base a los arts. 8 y $9 \mathrm{CEDH}$, la demandante del segundo caso se queja de la decisión por la que se le ha retirado la patria potestad y por la que se autorizó la adopción de su hijo.

El TEDH recuerda, en relación a ambos casos, los principios relativos a la infancia que ha establecido en la sentencia de la Gran Sala recaída en el caso Strand Lobben y otros c. Noruega, de 10 de septiembre de 2019. Cuando las autoridades se hacen cargo de un menor, estas mismas tienen la obligación de adoptar las medidas necesarias para facilitar la reunión de la familia a partir del momento en que sea posible.

A continuación, el TEDH subrayará que una vez que el interés de los padres entra en conflicto con el de los menores, las autoridades deben realizar un justo equilibrio. Solo en el caso de que se den circunstancias excepcionales puede romperse el vinculo familiar.

Los Estados disponen de un amplio margen de apreciación en la decisión de hacerse cargo de un menor, pero el TEDH debe ejercer un control más riguroso en cuanto a las restricciones complementarias, como las referidas al derecho de visita de los padres, que pueden comportar el riesgo de amputar las relaciones familiares entre los padres y el menor.

En el primer asunto, A.S. c. Noruega, el TEDH observa que, en su sentencia de marzo de 2015, por la cual se rechaza la solicitud de la demandante de poner fin al acogimiento de su hijo en una familia de acogida, el tribunal de distrito ha presumido que dicho acogimiento sería de larga duración. El procedimiento que ha precedido dicha decisión se ha basado en un acogimiento prolongado con la previsión de un régimen estricto de visitas.

El TEDH no ve razón alguna para poner en duda la apreciación del tribunal de distrito en relación con ciertos aspectos del asunto. No obstante, observa que es especialmente difícil concluir que la demandante presentaba lagunas en cuanto a aptitudes maternales elementales e intuitivas. Parece que se resuelve de esta manera en base a criterios vagos y subjetivos.

El TEDH subraya que la decisión del tribunal de distrito, que de hecho ha implicado el fin de la vida familiar de la demandante con su hijo, debió fundarse en una base fáctica suficiente y actualizada con la finalidad de dar respuesta adecuada a la alegación de la demandante de que sus aptitudes habían mejorado. 
El tribunal de distrito ha examinado un número de cuestiones pertinentes, pero ha rechazado todos los elementos a favor de la demandante con una motivación sucinta o inexistente. Así, su sentencia de marzo de 2015 se apoyó en viejos informes: las aptitudes maternales de la demandante fueron evaluadas por última vez de manera independiente en 2012 y son los padres de acogida los que han dado cuenta del desarrollo del niño entre 2013 y 2015, sin que sus observaciones fueran corroboradas por un tercero independiente.

La decisión por la que el tribunal ha rechazado poner fin al acogimiento se ha fundado en las reacciones negativas del menor durante los encuentros con su madre. Tales reacciones han sido señaladas en todo momento por los padres de acogida, que estaban con él en los encuentros con su madre. Los psicólogos han ofrecido informes divergentes en cuanto a las causas. En resumen, el tribunal de distrito ha motivado sucintamente su conclusión relativa a la naturaleza y a la causa de las reacciones negativas del niño.

El TEDH, subrayando la gravedad de la injerencia en causa y la importancia de los intereses en juego, considera que el proceso de decisión no ha tenido debidamente en cuenta los intereses de la demandante. Concluye que ha habido una violación del art. $8 \mathrm{CEDH}$.

En el asunto Abdi Ibrahim c. Noruega, el TEDH observa que la demandante no pretende que le devuelvan a su hijo, sino que no lo den en adopción. También impugna la eliminación de sus derechos parentales y la denegación de un derecho de visita. Incumbe a la Administración facilitar la vida familiar de la demandante y de su hijo, permitiéndoles al menos mantener una relación gracias a los contactos regulares organizados de una manera que sea compatible con el interés superior del niño.

El TEDH subraya que, desde el inicio, las autoridades internas han limitado de una manera sustancial los contactos entre la madre y su hijo, lo que en sí mismo comporta un riesgo de ruptura de los vínculos familiares.

El TEDH duda que la Administración haya adoptado las decisiones de acogimiento y adopción tras un control minucioso que permitiera concluir que la reunión familiar era imposible. Es más, dirá que ni la Administración ni los tribunales internos pueden utilizar una ruptura de relaciones familiares como motivo para autorizar la adopción cuando son ellos mismos los que han creado la situación incumpliendo su obligación de tomar las medidas adecuadas para reunificar a la familia.

Uno de los elementos principales que el tribunal de apelación ha considerado es la reacción negativa del niño en los encuentros con su madre. No obstante, no puede extraerse de ello conclusiones claras en cuanto a los futuros encuentros. El tribunal de apelación ha concluido sucintamente en cuanto a la causa de las reacciones negativas del niño durante los encuentros, lo que ha servido para motivar la decisión de autorizar la adopción. No había elementos suficientes para pensar que los contactos entre la madre y el niño serían siempre negativos hasta el punto de concluir que la ruptura de todo vínculo con la demandante constituía el interés superior del menor. 
En fin, el tribunal de apelación además ha dado más importancia al perjuicio que el menor sufriría si se separase de sus padres de acogida que a los motivos que justifican la ruptura de toda relación con su madre. Parece que ha pesado más la oposición de los padres de acogida a una «adopción abierta» que habría permitido a la demandante continuar en contacto con su hijo, que el interés de esta última a seguir una vida familiar con su hijo.

El TEDH concluye que las autoridades no han dado suficiente importancia al derecho de la demandante y de su hijo de gozar de una vida familiar, tanto en un sentido general como específico, especialmente en cuanto a las razones de tipo cultural y religioso.

El TEDH subraya la gravedad de la injerencia en este caso y la importancia de los intereses en juego en el proceso de decisión, que ha conducido a la retirada de la patria potestad de la demandante y a la adopción del menor, lo cual se ha realizado sin tener debidamente en cuenta todos los pareceres e intereses de la demandante.

Por todo ello, el TEDH concluye que ha habido una violación del art. 8 $\mathrm{CEDH}$.

En la sentencia recaída en el caso K.O. y V.M. c. Noruega, de 19 de noviembre de 2019, los demandantes son una pareja de ciudadanos noruegos nacidos en 1974 y 1986 respectivamente. Al dar a luz a una nińa en enero de 2015, los servicios de protección de la infancia, preocupados por la salud mental de la Sra. V.M., su adicción a las drogas y los conflictos conyugales con el Sr. K.O., organizaron la estancia de la madre y su hija en un centro familiar. Parece que unas semanas más tarde la demandante retiró el consentimiento a su estancia en dicho centro. Los servicios de protección de la infancia decidieron hacerse cargo de la menor por razones de urgencia. Solicitaron la puesta de la menor en una familia de acogida. En mayo de 2015, la oficina de ayuda social, compuesta por un jurista habilitado a realizar la función de juez, dos psicólogos y dos asesores no profesionales, estimaron la solicitud, una vez oídos once testigos, en presencia de los padres y de sus abogados. Concluyó que el interés superior del menor exigía la entrega de la menor a una familia de acogida.

Si bien los demandantes recurrieron en la vía interna, no tuvieron éxito. El tribunal de distrito, en concreto, dijo que la menor estaba en riesgo si continuaba viviendo con sus progenitores debido a los problemas antes mencionados. Subrayó que los intentos llevados a cabo para solucionar sus dificultades fueron infructuosos y que los interesados no cooperaron con los servicios sociales. Indicó que el padre había sido igualmente condenado por infracciones graves, en concreto, por delitos de violencia y amenazas.

Tanto la oficina de ayuda social como el tribunal de distrito consideraron que la entrega en acogimiento sería de larga duración y que no era del interés 
superior del menor reconocer un amplio derecho de visita a los padres. Por ello, se establecerían seis visitas anuales de dos horas.

La niña fue finalmente devuelta a sus padres a raíz de la decisión de levantamiento de la medida adoptada en marzo de 2008 por el tribunal de distrito. Los expertos habrían informado favorablemente acerca de la aptitud de los padres para hacerse cargo de su hija. Su situación era buena y estable y habían aceptado las medidas de asistencia.

Agotada la vía interna, los demandantes acuden ante el TEDH alegando una violación del art. $8 \mathrm{CEDH}$ (derecho al respeto de la vida privada y familiar). Consideran que el derecho de visita acordado fue muy restrictivo.

El TEDH considera que el proceso en la toma de la decisión de dar en acogida a la menor, tanto ante el órgano de ayuda social como ante el tribunal de distrito, ha sido meticuloso y los demandantes han podido hacer valer plenamente sus argumentos. Los interesados han podido presentar una solicitud de levantamiento de la medida doce meses después. El proceso nacional ha protegido suficientemente sus derechos.

El TEDH está convencido de que las autoridades nacionales han realizado un examen en profundidad del asunto teniendo en cuenta los antecedentes de los demandantes y valorando la adopción de medidas menos radicales. Han llegado, no obstante, a la conclusión de que ninguna otra medida era viable teniendo en cuenta que se había intentado ayudar a los demandantes por diferentes vías y la dificultad de estos últimos de cooperar con los servicios de protección de la infancia.

En suma, el TEDH considera que las razones ofrecidas para dar en acogida a la menor han sido pertinentes y suficientes y que la injerencia en el derecho al respeto de la vida familiar de los demandantes no ha sido desproporcionada. No ha habido, por tanto, una violación del art. $8 \mathrm{CEDH}$.

Por el contrario, la oficina de ayuda social y el tribunal de distrito no han considerado en ningún momento, de una manera seria, la reunificación familiar en sus decisiones relativas al derecho de visita de los demandantes y, al considerar que la acogida sería de larga duración, han descartado implícitamente esta posibilidad desde muy temprano.

El TEDH subraya que si existe una perspectiva de reunir a la familia en el futuro no se pueden dar espacios de tiempo tan prolongados de semanas o incluso meses entre visita y visita. Las decisiones relativas al derecho de visita han tenido como única finalidad que la niña pudiera conocer quiénes son sus padres biológicos.

Las autoridades internas no han logrado justificar en qué medida impedir visitas más frecuentes atendía al interés superior de la menor, habida cuenta de que las visitas de sus padres tenían efectos positivos en ella. El TEDH concluye que ha habido una violación del art. $8 \mathrm{CEDH}$ en lo que concierne a las restricciones en las visitas. 


\section{GESTACIÓN SUBROGADA}

En la Decisión de Inadmisión recaída en el caso Petithory Lanzmann c. Francia, de 5 de diciembre de 2019, la demandante es la viuda de Claude Lanzmann, escritor y director de la película Shoah, que falleció en 2018. Su hijo murió el 13 de enero de 2017 debido a un tumor cancerígeno diagnosticado en 2014. Tan pronto como fue informado de su enfermedad, su hijo expresó su deseo de ser padre y tener sucesión, incluso en caso de muerte. Por ello, depositó esperma en el Centre d'études et de conservation des oeufs et du sperme (CECOS) del Hospital Cochin de París, contactó con un centro en Suiza y consideró otras opciones para el almacenamiento de su esperma en bancos de semen, pero no pudo realizar estas últimas opciones debido a su enfermedad.

En la primavera de 2017 el presidente del CECOS rechazó remitir a la Agencia de Biomedicina la solicitud del demandante de transferencia de su esperma a un establecimiento de salud de Israel. La demandante solicitaría entonces al Tribunal Administrativo de París, por la vía de urgencia, la autorización de dicha transferencia. Argumentó que la denegación de la solicitud le privó de su derecho a ejercer su vida privada y familiar, ya que aspiraba a ser abuela y a que se respetaran los deseos de su hijo. El juez que tramita las solicitudes de urgencia rechazó su demanda. La demandante recurrió contra la decisión ante el Consejo de Estado, sede en la que también se rechazarían sus pretensiones.

Agotada la vía interna, la demandante acude ante el TEDH alegando una violación del art. $8 \mathrm{CEDH}$. La demandante dice que las autoridades internas no le han permitido acceder al esperma de su hijo, con el que podría haber llegado a un acuerdo, de conformidad con sus últimas voluntades, de reproducción médica asistida para una pareja infértil o de gestación subrogada, procedimientos que serían autorizados en Israel o en los Estados Unidos de América.

El TEDH observa que la queja de la demandante comprende dos partes. En la primera dice que es una víctima indirecta, al intervenir en el proceso en nombre de su hijo. En la segunda, alega que es una víctima directa, ya que ha sido privada de la posibilidad de ser abuela.

En relación con su consideración como víctima indirecta, el TEDH observa que los derechos alegados por la demandante se refieren al derecho de su hijo a decidir cómo y cuándo ser padre. Sin embargo, el Tribunal observa que sus derechos pertenecen a la categoría de derechos no transferibles. Por ello, la demandante no podría reclamar a este respecto ser una víctima de una violación del Convenio en el nombre de su hijo.

En relación con la queja alegando el estatus de víctima directa, el TEDH considera que el art. $8 \mathrm{CEDH}$ no garantiza un derecho a ser abuelo, bien que la aspiración de la demandante de continuar con su línea genética es legítima.

Por todo ello, el TEDH inadmite la demanda. 
Dos nuevos casos llegan al TEDH sobre el tema de la inscripción en Francia de nacimientos por gestación subrogada en terceros países. Me refiero a la Decisión de Inadmisión C. y E.c. Francia, de 12 de diciembre de 2019.

La demanda en el primer caso - C. c. Francia - se presenta por tres ciudadanos franceses: una pareja —el Sr. y la Sra. C. - y un menor.

El niño nació en febrero de 2010 en los Estados Unidos de América de gametos del Sr. C. y de una tercera donante. La partida de nacimiento, de octubre de 2010, indica que la Sra. C. es la madre y que el Sr. C. es el padre.

Los esposos solicitaron, en 2014, la transcripción de la partida de nacimiento en los registros civiles del consulado francés de Miami. Esta solicitud fue remitida a la fiscalía de Nantes al sospechar que se trataba de un niño concebido por gestación subrogada. Los demandantes recibieron la notificación de la desestimación de su solicitud en 2015. Entonces acudieron ante el Tribunal de Gran Instancia de Nantes alegando una violación del art. $8 \mathrm{CEDH}$ (derecho al respeto de la vida privada y familiar) y $14 \mathrm{CEDH}$ (interdicción de la arbitrariedad). Pedían que se transcribiera la partida de nacimiento de su hijo en los registros civiles de Francia. Y efectivamente el tribunal de gran instancia dio la razón a los demandantes. No obstante, en apelación solo se permitiría la inscripción de la paternidad del Sr. C., no así la maternidad de su esposa, al no haber dado a luz al menor.

En el segundo caso, E. c. Francia, la demanda se presenta por cinco ciudadanos franceses: el Sr. y la Sra E. y tres menores nacidos en 2014.

Los tres niños nacieron en Ghana, de gametos del Sr. E. y de una tercera donante. La familia se estableció en ese país. La partida de nacimiento indica que la Sra. E. es la madre de los tres niños y que el Sr. E. es su padre. Solicitaron la transcripción de las partidas de nacimiento a la embajada de Francia en Ghana.

El fiscal de Nantes informó a los esposos en 2014 que los niños habían nacido de un contrato de gestación subrogada, lo que está prohibido por el art. 16.7 del CC francés, por lo que decidió suspender la transcripción de las actas de nacimiento a la espera de instrucciones de la cancillería. Si bien el tribunal de gran instancia y el tribunal de apelación accederían a la transcripción de las partidas de nacimiento, el tribunal de casación, finalmente, anuló lo referido a la maternidad de la Sra. E., al no haber concebido a los menores, manteniéndose, sin embargo, la paternidad del Sr. E.

Ante el TEDH, los demandantes en ambos casos alegan una violación del art. $8 \mathrm{CEDH}$ porque las autoridades francesas se negaron a la transcripción de la integridad de las partidas de nacimiento. En base al art. 14 (prohibición de la discriminación), combinado con el art. $8 \mathrm{CEDH}$, los demandantes denuncian una discriminación en su derecho al respeto de la vida privada de los menores.

El TEDH recuerda que el 10 de abril de 2019 ha dictado una opinión consultiva sobre la situación en la que un menor ha nacido en el extranjero por gestación subrogada de gametos de un padre de intención y un tercer donante y en el que el vínculo de filiación ente el niño y su padre de intención ha sido reconocido en derecho interno. 
El TEDH observa que la situación de los niños demandantes corresponde a este caso. El derecho interno ofrece una posibilidad de reconocimiento del vínculo de filiación entre los niños demandantes y su madre de intención por la vía de la adopción. Así, remite a sentencias del tribunal de casación de 5 de julio de 2017 y no considera que haya razones para desconfiar del Gobierno. El TEDH observa que el procedimiento de adopción con la finalidad del reconocimiento del vínculo de filiación no es una carga excesiva. El TEDH observa que la Administración resuelve estos procesos en unos cuatro meses.

Por consiguiente, el TEDH concluye que la denegación de las autoridades francesas de la transcripción de las partidas de nacimiento extranjeras de menores en los registros civiles franceses en cuanto a la designación de la madre de intención como su madre no ha sido desproporcionada en relación con los fines perseguidos, por lo que inadmite la demanda a este respecto.

En cuanto a la alegación de una violación del art. 14 combinado con el art. 8, el TEDH precisa que la diferencia entre «los otros menores nacidos en el extranjero" y «los menores nacidos de una gestación subrogada en el extranjero» consiste únicamente en que los nacidos de una gestación subrogada no pueden obtener una transcripción integral de la partida de nacimiento extranjera y deben pasar por la vía de la adopción. Observa que se deriva de las explicaciones del Gobierno que esta diferencia de trato permite conocer las circunstancias concretas de cada caso y salvaguardar el interés del menor nacido de una gestación subrogada con la finalidad de confirmar que tal vínculo se ha establecido con respecto de la madre de intención. Recuerda también que ha establecido en su opinión consultiva de 10 de abril de 2019 que la elección de los medios para ofrecer el reconocimiento del vínculo entre menores y padres de intención corresponde al margen de apreciación de los Estados y que el art. $8 \mathrm{CEDH}$ no establece una obligación general de reconocer desde el inicio un vínculo de filiación entre el menor y la madre de intención. Por consiguiente, la diferencia de trato denunciada atiende a una justificación objetiva y razonable. Por ello, el TEDH inadmite también esta parte de la demanda.

\section{DERECHO AL RESPETO DE LOS BIENES}

\section{SANIDAD ANIMAL: NO INDEMNIZACIÓN POR LA ORDEN DE SACRIFICIO DE ANIMALES ENFERMOS}

En la sentencia recaída en el caso S. A. Bio d'Ardennes c. Bélgica, de 12 de noviembre de 2019, la demandante es una sociedad limitada belga. Su ámbito de actividad comercial se refiere a la producción de cárnicos, en concreto, de ternera.

La empresa compró, a través de la intermediación de la asociación DGZ, 27 cabezas de ganado de origen portugués en julio de 1998 y después 62 más en agosto de 1998 y las colocó en sus granjas. 
En marzo de 2000 el inspector de veterinaria notificó a la empresa un brote de brucelosis en una de sus manadas y se le ordenó el sacrificio de los animales afectados. La empresa sacrificó entonces 118 cabezas de ganado el 22 de marzo de 2000. El mismo día, se detectó otro brote de brucelosis, por lo que se notificó otra orden de sacrificio y se ejecutó. Finalmente, otras 76 cabezas de ganado fueron incautadas y sacrificadas el 28 de abril de 2000, en base a la Ley de 24 de marzo de 1987.

La Administración rechazó la solicitud de indemnización realizada por la empresa en base a los sacrificios de las cabezas de ganado que debió acometer. La Administración consideró que la empresa había cometido numerosas infracciones que resultaron o pudieron haber resultado en la propagación de la contaminación de la totalidad de su ganado.

El TEDH observa que el Real Decreto de 6 de diciembre de 1978 prevé, en principio, una indemnización parcial por el sacrificio de ganado afectado por brucelosis. Sin embargo, no se le ha reconocido la indemnización a la empresa demandante debido a la comisión de infracciones contempladas en la normativa aplicable. La denegación de la indemnización, en el caso de cometer dichas infracciones, está prevista expresamente en el art. 23.3 del Real Decreto citado. La empresa demandante no habría alegado que no tuviera conocimiento de las obligaciones establecidas en la normativa o que no había cometido las infracciones en cuestión.

En segundo lugar, los tribunales internos han verificado que las condiciones que justifican la interferencia en los derechos de propiedad, tal y como las interpreta el TEDH, se han satisfecho. El TEDH observa que el razonamiento de los tribunales internos no es arbitrario ni manifiestamente irrazonable.

En tercer lugar, el TEDH observa que la empresa ha obtenido una compensación por 89 cabezas de ganado sacrificadas por la negligencia de la asociación DGZ - la empresa demandante habría alegado que esta asociación no le ofreció la información adecuada, pues, en otro caso, no habría adquirido el ganado- - y tuvo en cuenta este hecho para realizar la evaluación de la proporcionalidad de las medidas.

En cuarto lugar, el TEDH constata que en el ordenamiento belga hay normas que sancionan el incumplimiento de la normativa sanitaria reduciendo el derecho de indemnización en lugar de excluirlo. Esto no es apto, continúa el TEDH, en este caso para cuestionar el juicio de ponderación realizado entre la protección de la propiedad y los requerimientos del interés general. Las autoridades internas tienen un margen de discreción en el ámbito de la protección de la salud pública en su territorio y en la determinación de las sanciones por infracciones de la normativa sanitaria, dependiendo de los riesgos que se dan del incumplimiento y de la naturaleza de las enfermedades de los animales.

Finalmente, la empresa demandante ha podido continuar sus actividades adquiriendo nuevas cabezas de ganado una vez que las medidas sanitarias se han levantado y no ha dicho que esto haya sido imposible o excesivamente difícil. 
En consecuencia, el Tribunal sostiene que, a la vista de la importancia que tiene para los Estados la prevención de las enfermedades animales y del margen de apreciación que se les ofrece a este respecto, la empresa demandante no ha tenido que afrontar una carga excesiva por la denegación de la indemnización por el sacrificio de su ganado. Por ello, el TEDH considera que no ha habido una violación del art. 1 del Protocolo adicional núm. 1 al Convenio.

\section{VÍA DE HECHO}

En la sentencia recaída en el caso Khizanishvili y Kandelaki c. Georgia, de 17 de diciembre de 2019, el Ayuntamiento de Tiflis derriba el edificio de los demandantes sin que se haya seguido un procedimiento administrativo. La prensa dijo que se trató de una demolición llevada a cabo en el marco de un plan de eliminación de todos los edificios construidos ilegalmente o antiestéticos.

Los tribunales internos reconocieron la ilegalidad de la demolición. El tribunal de primera instancia ordenó indemnizar al primer demandante con 80.000 euros y al segundo con 62.000, tras un cálculo basado en el valor de mercado del bien y excluyendo el valor del terreno que los demandantes habían conservado.

En apelación, la indemnización se redujo a aproximadamente 1.100 euros para el primer demandante y 617 para el segundo demandante. Este nuevo cálculo se basó en una evaluación del valor de mercado de los escombros. Los demandantes recurrieron ante el Tribunal Supremo, pero su demanda se inadmitiría en esta sede.

Agotada la vía interna, los demandantes acuden ante el TEDH alegando una violación del art. 1 del Protocolo adicional núm. 1 al Convenio. Sostienen que la indemnización que se les otorgó no correspondía con las pérdidas reales sufridas por la demolición ilegal de su bien.

Aunque los demandantes no hayan sido privados de su título de propiedad sobre la parcela del terreno sobre la cual se situaba el inmueble destruido, la demolición en sí misma debe analizarse como una expropiación de facto.

Las jurisdicciones internas han constatado que dicha demolición no tenía base legal alguna. La injerencia no ha sido legal ni perseguía un fin legítimo de utilidad pública. Las jurisdicciones nacionales no han invocado, en efecto, ninguna utilidad pública ni en la vía interna ni ante el TEDH.

La cuestión central será determinar si la indemnización otorgada a los interesados fue suficiente.

Para reducir las sumas reconocidas en primera instancia, el tribunal de apelación se basa en el valor del material obtenido tras la demolición. Dice que es conforme a la manera en la que el valor del bien era habitualmente calculado en asuntos similares. No se ha referido, sin embargo, a ningún otro asunto ni ha expuesto en qué eran comparables dichos asuntos. No ha ejercido el derecho que le ofrece la ley de solicitar otro informe para clarificar estas cuestiones. 
Además, el tribunal de apelación no ha valorado si el método de cálculo y, sobre todo, las indemnizaciones que resultaron, eran equitativas y suficientes a la vista de la diferencia evidente entre el valor del inmueble habitado y el de los materiales resultantes de la demolición.

Por todo ello, los demandantes no han obtenido la reparación integral que razonablemente podían esperar teniendo en cuenta el atentado grave a su derecho de propiedad, por lo que ha habido una violación del art. 1 del Protocolo adicional núm. 1.

\section{DERECHO A LA INSTRUCCIÓN}

En la sentencia recaída en el caso Papageorgiou y otros c. Grecia, de 31 de octubre de 2019, los demandantes son cinco ciudadanos griegos, padres y sus hijos, que residen en las pequeńas islas griegas de Milos y Sifnos. Los tres primeros demandantes son Petros Papageorgiou y Ekaterini Berdologlou y la hija de ambos. El cuarto y quinto demandantes son Rodopi Anastasiadou y su hija Smaragda Raviolou.

En virtud de la Constitución de Grecia, así como de otros textos legislativos, como la Ley de educación y diversos reglamentos ministeriales, la educación religiosa es obligatoria para todos los alumnos de la enseńanza primaria y secundaria.

Los demandantes solicitaron al Consejo de Estado, en julio de 2017, la anulación de dos reglamentos ministeriales recientes que establecían el programa de educación religiosa para el curso escolar 2017/2018. En ese momento, Maria Rafaella Papageorgiou estaba terminando sus estudios de enseńanza general, mientras que Smaragada Raviolouestaba se encontraba en cuarto año de la escuela primaria de Sifnos.

Los demandantes solicitaron que su asunto fuera examinado con carácter de urgencia antes del inicio del curso escolar, pero el Consejo de Estado rechazó sus demandas, pues consideró que no tenían una importancia suficiente.

El Consejo de Estado no ha resuelto todavía el asunto. La primera audiencia se fijó para septiembre de 2018, es decir, después de la finalización del curso escolar concernido.

Ante el TEDH, los padres alegan que, en la solicitud de dispensa de la educación religiosa, es necesario declarar que no son cristianos ortodoxos. Añaden que el director del centro escolar debió verificar la veracidad de sus declaraciones y que las declaraciones de este tipo se conservan en los archivos de la escuela. Invocan, en concreto, el art. $9 \mathrm{CEDH}$ (liberad de pensamiento, de conciencia y de religión) y el art. 2 del Protocolo núm. 1 (derecho a la instrucción) CEDH.

El TEDH decide examinar la demanda desde la perspectiva del art. 2 del Protocolo núm. 1 del CEDH, que otorga a los padres el derecho a exigir al Estado 
el respeto de sus convicciones religiosas y filosóficas en la enseñanza del hecho religioso. Interpreta también esta disposición a la luz del art. $9 \mathrm{CEDH}$, que garantiza a los alumnos el derecho a la instrucción en armonía con el derecho a creer o a no creer.

En primer lugar, el TEDH considera que el principal problema que subyace a este asunto se refiere a que si los padres quieren dispensar a sus hijos del curso de religión, están obligados a declarar solemnemente que sus hijos no son cristianos ortodoxos. Los mecanismos de dispensa -o la posibilidad de asistir a un curso alternativo- existen en la casi totalidad de los Estados del Consejo de Europa. El TEDH estima que es importante saber si las condiciones de exención o de derogación implican una carga indebida a los padres, por ejemplo, al obligarles a comunicar sus convicciones religiosas o filosóficas. El TEDH observa que los padres demandantes debieron realizar una declaración que permitía deducir que tanto ellos como sus hijos no pertenecían a una religión cristiana ortodoxa. De hecho, el sistema actual de dispensa de educación religiosa para los alumnos griegos supone un riesgo de divulgación de aspectos sensibles de la vida privada. Este sistema puede disuadir la solicitud de la dispensa, pues supone que el director del establecimiento verificará las informaciones que figuran en la declaración.

El conflicto potencial se acentúa en el caso de los demandantes, pues viven en pequeñas islas en las que la inmensa mayoría de la población se identifica con una religión dada, por lo que el riesgo de estigmatización es mayor que en las grandes ciudades. Además, como observan los demandantes, los alumnos dispensados en realidad no tenían la posibilidad de cursar una asignatura alternativa, con lo que habrían perdido horas de clase solo por haber revelado sus creencias.

En fin, como las autoridades no tienen derecho a intervenir en el ámbito de la conciencia del individuo, a verificar las creencias religiosas de las personas u obligarles a desvelarlas, el TEDH concluye que ha habido una violación del art. 2 del Protocolo núm. 1, interpretado a la luz del art. 9 del Convenio ${ }^{2}$.

2 Sobre este tema, desde una perspectiva de los derechos nacionales y supranacionales, me remito a L. Martín-Retortillo Baquer (2008), Los padres tendrán derecho preferente a escoger el tipo de educación que habrá de darsea sus hijos (Un estudio de jurisprudencia del Tribunal Europeo de Derechos Humanos), Zaragoza: El Justicia de Aragón; P. Meix Cereceda y J. de Groof (coords.) (2017), Religious and ideological rights in education. Judicial perspectives from 32 legal systems, Oisterwik: Wolf Legal Publishers; P. Meix Cereceda (2017), «El Tribunal Europeo de Derechos Humanos y las convicciones particulares en la escuela: entre el "interés del menor» y el "margen de apreciación" de los Estados", Academia: revista sobre enseñanza del derecho de Buenos Aires, 29, págs. 197-214; y P. Meix Cereceda (2014), El derecho a la educación en el sistema internacional y europeo, Valencia: Tirant lo Blanch. 


\section{NON BIS IN IDEM}

En la sentencia recaída en el caso Mihalache c. Rumanía, de 8 de julio de 2019 , el demandante dio positivo en un control de alcoholemia. Fue requerido por los agentes de policía a acompañarle para realizar un análisis con la finalidad de determinar el nivel de alcohol en sangre, pero se negó. Se inició entonces un proceso penal contra él. Un mes después el fiscal daría por finalizado el proceso al considerar que los actos cometidos no fueron suficientemente graves para constituir un delito. Sin embargo, le impuso una multa administrativa equivalente a unos 250 euros. El demandante no recurrió y la pagó.

Unos meses después, un superior jerárquico del fiscal que dio por finalizado el procedimiento penal decidió de oficio la anulación de esa decisión al considerar que la multa administrativa no fue apropiada a la luz del grado de peligro general y específico que implicaban los hechos del caso. En consecuencia, se devolvió el caso a la fiscalía con la finalidad de que continuara la investigación penal.

El demandante finalmente fue condenado a una pena suspendida de un ańo de prisión por haberse negado a realizar el análisis de sangre. Recurrió la decisión, pero el tribunal de apelación desestimó.

El fiscal jefe requirió a las autoridades financieras la devolución de la cantidad dineraria correspondiente a la multa. Así, un agente de policía se personó en el domicilio del demandante con la finalidad de informarle sobre el procedimiento de devolución de la multa. Según los documentos que constan en el expediente, el demandante en ningún momento solicitó el reintegro de las cantidades pagadas.

En base al art. 4 del Protocolo núm. 7 el demandante se queja de que ha sido procesado y sancionado dos veces por la misma infracción. También alega que la reapertura del proceso contra él no respeta los criterios establecidos en este precepto.

El TEDH subraya que el principio non bis in idem comprende tres elementos.

En primer lugar, ambos procedimientos deben ser penales in natura. En el presente caso, el TEDH concluyó que ambos procesos - el resultante en la orden de 7 de agosto de 2008 por el que se impuso la multa administrativa y el resultante de la sentencia de 14 de junio de 2010 del tribunal de apelación por el que se dictó una pena de prisión suspendida- lo eran.

En segundo lugar, ambos procesos deben referirse a los mismos hechos. El TEDH observa que el demandante, en ambos casos, ha sido procesado y sancionado por negarse a la práctica del análisis de sangre tras un control de tráfico. Las dos decisiones, por consiguiente, se referían a los mismos hechos y los mismos cargos.

En tercer lugar, debe darse una duplicación de procedimientos. El TEDH subraya que el art. 4 del Protocolo núm. 7 tiene como finalidad la prohibición de la duplicación de procedimientos penales con una finalidad íntimamente vinculada. El TEDH observa que la orden de 7 de agosto de 2008, por la que se le impone una multa de unos 250 euros, constituyó una condena (una sanción 
punitiva), que devino firme, en el sentido del Convenio, en el plazo de veinte días establecido en el art. 249 del Código Procesal Penal de Rumanía, en vigor en aquel momento. Así, el TEDH señala que la única opción que contemplaba ese precepto era un recurso ordinario para determinar si la orden de 7 de agosto había ganado firmeza, no así la anulación por parte del superior jerárquico de la decisión del fiscal de rango inferior (arts. 270 y 273 del Código). Debido a la ausencia de un plazo, el derecho rumano no regula de una manera suficientemente clara la manera en la que el recurso debe utilizarse, lo que ha causado inseguridad jurídica en el demandante.

Dado que la orden de pago de la multa devino firme en el sentido del Convenio, su anulación por el fiscal superior y la reapertura del proceso constituyó una duplicación del proceso. Esta duplicación puede ser compatible con el Convenio si el segundo proceso constituye una reapertura conforme a las condiciones del art. 4, Protocolo $7^{\circ}$. En este caso el TEDH observa que el fiscal de rango superior ha decidido en base a un mismo expediente del caso sin que se haya dado ningún hecho nuevo o por algún error fundamental en el proceso previo. El TEDH considera, por consiguiente, que las razones expuestas por el fiscal superior para justificar la reapertura del proceso no respetaron los criterios estrictos establecidos por el art. 4 del Protocolo núm. 7, por lo que no se ha justificado la reapertura del proceso.

El TEDH concluye, en fin, que el demandante ha sido juzgado dos veces por la misma infracción, en violación del principio non bis in idem. Ha habido, por tanto, una violación del art. 4 del Protocolo núm. 7 del Convenio 3 .

3 Véase, al respecto, T. Cano Campos (2001), «Non bis in idem, prevalencia de la vía penal y teoría de los concursos en el Derecho administrativo sancionador», RAP, 156, págs. 191-250. 
